\title{
Instantaneous Actual Motion Estimation with a Single High-Resolution Radar Sensor
}

Johannes Schlichenmaier*, Leping Yan*, Martin Stolz ${ }^{\dagger}$, and Christian Waldschmidt* *Institute of Microwave Engineering, Ulm University, 89081 Ulm, Germany Email: johannes.schlichenmaier@uni-ulm.de

${ }^{\dagger}$ Robert Bosch GmbH, Advanced Engineering Sensor Systems, P.O. Box 16 61, 71226 Leonberg, Germany 


\title{
Instantaneous Actual Motion Estimation with a Single High-Resolution Radar Sensor
}

\author{
Johannes Schlichenmaier*, Leping Yan*, Martin Stolz ${ }^{\dagger}$, and Christian Waldschmidt* \\ ${ }^{*}$ Institute of Microwave Engineering, Ulm University, 89081 Ulm, Germany \\ Email: johannes.schlichenmaier@uni-ulm.de \\ ${ }^{\dagger}$ Robert Bosch GmbH, Advanced Engineering Sensor Systems, P.O. Box 16 61, 71226 Leonberg, Germany
}

\begin{abstract}
Future high-resolution radars enable new functionalities in advanced driver assistance systems, relying on fast and reliable extraction of properties of vehicles on the road. A critical property for the prediction of trajectories and the assessment of potentially dangerous situations is that of the actual motion - the velocity vector and yaw rate - of observed objects. In this paper, an approach to distinguish linear from non-linear motions as well as estimating the yaw rate using only a single radar sensor is presented and evaluated via measurements.
\end{abstract}

\section{INTRODUCTION}

Future applications of automotive radar, like advanced driver assistance systems (ADAS) and autonomous driving heavily rely on the ability to extract various properties from extended objects like vehicles on the road. Aside from static properties like length, width, position, and orientation, the object's actual motion is of very high interest to the prediction of trajectories. For linear motion, actual motion of a vehicle is described by its velocity vectors $v_{\mathrm{x}}$ and $v_{\mathrm{y}}$, for nonlinear motion by its yaw rate $\omega$ and instantaneous center of rotation (ICR).

Usually, actual motion estimation by radar is achieved by using two sensors [1] or restricting the detection to linear motion [2]. Another solution would be that of tracking [3], [4], although tracking solutions are never instantaneous and require multiple tracking steps to reliably extract the desired properties from the extended object.

In many instances however, measurements from only a single sensor are available, be it because the system is only equipped with one sensor, or, because the fields of view (FoVs) of the installed sensors do not, or only partially, overlap.

In these situations, vehicle properties like length, width, position, and orientation may still be extracted, either by constructing an oriented bounding box (OBB) from the clustered target points [5], or by searching for a template-match within the target points [6]. A complete actual motion estimation however is not possible from the target points alone.

In this paper, an approach for estimating the actual motion in linear and nonlinear scenarios based on the measurements of a single radar sensor is presented. For this, it makes use of the extracted contour in form of an OBB.

\section{Problem Formulation}

For a sensor at position $(0,0)$, the basis for obtaining the vehicles actual motion parameters is the relation between the radial velocity $v_{\mathrm{r}}$ and angle of arrival $\theta$ of a target point $i$, and

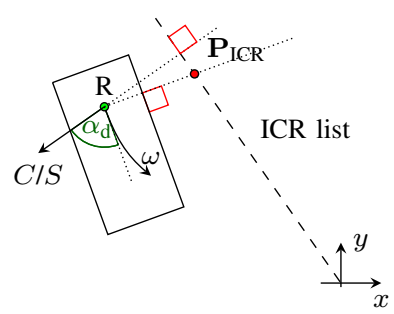

(a) Nonlinear motion

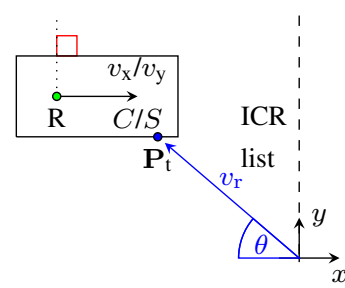

(b) Linear motion.
Fig. 1. Geometric relations between contour orientation, reference points and the motion parameters of objects.

the vehicle's velocity vector for linear (Eq. 1), and the yaw rate and ICR $x$ - and $y$-values for nonlinear motion (Eq. 2).

$$
\begin{array}{lll}
v_{\mathrm{r}, i}=v_{\mathrm{x}} & \cos \left(\theta_{\mathrm{i}}\right)+v_{\mathrm{y}} & \sin \left(\theta_{\mathrm{i}}\right) \\
v_{\mathrm{r}, i}=\underbrace{\omega y_{\mathrm{ICR}}}_{\mathrm{C}} & \cos \left(\theta_{\mathrm{i}}\right)+\underbrace{\left(-\omega x_{\mathrm{ICR}}\right)}_{\mathrm{S}} & \sin \left(\theta_{i}\right)
\end{array}
$$

The vehicle's velocity profile parameters $C$ and $S$ are extracted via a velocity profile analysis (VPA). This is done by analyzing the target points' $v_{\mathrm{r}}$ over $\theta$ using a random sample consensus (RANSAC) algorithm - which additionally divides the target points into the ones laying inside the vehicle's bulk motion corridor and the ones not - and a subsequent orthogonal regression optimization [2].

For linear motions, $C$ and $S$ can be directly assigned to $v_{\mathrm{x}}$ and $v_{\mathrm{y}}$, respectively. For nonlinear motions however, the parameters are comprised of two components, the ICR and the yaw rate $\omega$.

Therefore the proposed algorithm has to be able to distinguish between linear and nonlinear motion, and then separate the yaw rate and ICR position for the nonlinear motion.

\section{LiNEAR AND NONLINEAR MOTION}

In Fig. 1, the geometric relations between a vehicle's contour, its orientation, the reference point (generally placed on the middle of the rear axle) and the extracted velocity profile parameters $C$ and $S$ are depicted.

The straight line annotated as ICR list is generated by solving $S$ for $x_{\mathrm{ICR}}$ and $C$ for $y_{\mathrm{ICR}}$, and choosing different values for $\omega$ from $-\infty \rightarrow 0$ and $\infty \rightarrow 0$. It is always orthogonal to the vector spanned by $C$ and $S$. 


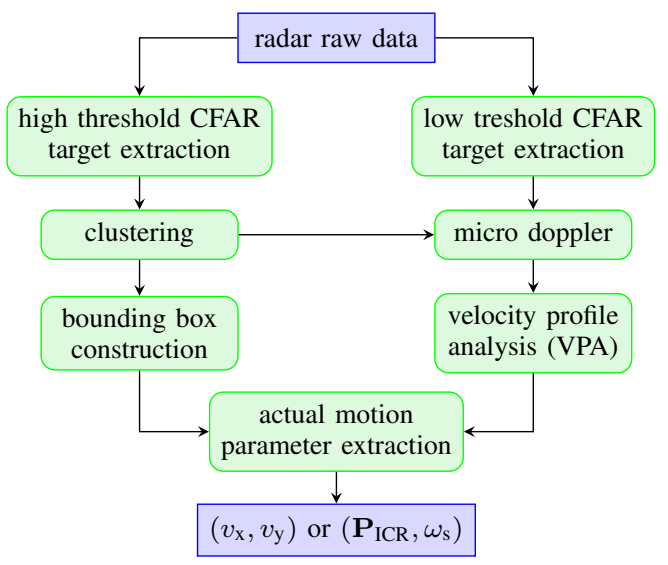

Fig. 2. Algorithm chain for single sensor actual motion estimation.

For nonlinear motion (Fig. 1a), the vehicle turns around the point $\mathbf{P}_{\text {ICR }}$ with the yaw rate $\omega$, which is at the intersection of the ICR list and the line perpendicular to the vehicles orientation originating from the vehicle's reference point.

For linear motion (Fig. 1b), the $C / S$ vector is identical with the $v_{\mathrm{x}} / v_{\mathrm{y}}$ vector and the ICR list is perpendicular to the vehicles orientation. There now is no intersection between the ICR list and the line originating from the reference point, resulting in $\omega=0$ and a ICR point at infinity.

This shows, that if a good estimation of the contour and the velocity profile parameters $C$ and $S$ can be achieved, both can be used to discriminate between linear and nonlinear motion on the one hand, and - in the case of nonlinear motion to separate the parameters $C$ and $S$ on the other hand, thus allowing for an estimation the yaw rate of the vehicle.

\section{Algorithm Chain}

The algorithm chain used for achieving the aforementioned goals is shown in Fig. 2.

It starts by taking the radar raw data before the direction of arrival (DoA) estimation - in the case of a chirp sequence modulated radar, the range-velocity-domain - and feeds it to two different algorithm chains.

In the first chain, the contour of the vehicle is estimated. For this, it performs constant false alarm rate (CFAR) algorithm with a high threshold followed by a peak search to extract only strong reflections. For those, a DoA estimation is performed, after which the resulting target points are clustered spatially, and for each cluster, an OBB is constructed.

In parallel, the second algorithm chain also performs a target extraction and DoA estimation, but this time, the CFAR threshold is very low and no peak search is performed. This allows also for very weak reflections to be considered, as well as the micro Doppler information of extended targets, which is hidden in the side bands of the peaks.

A clustering of the low-CFAR target points is not advisable, because of the presence of low power clutter and noise, making building correct clusters very difficult. Instead, the clustering information from the first chain is used to extract microDoppler reflections in and around the extended object.
With the extracted micro-Doppler reflections, a velocity profile analysis (VPA) is performed as previously described, which then yields the velocity profile parameters $C$ and $S$. Those, together with the contour from the first chain, are then fed into the actual motion parameter extraction subroutine.

\section{Actual Motion Parameter Extraction}

As was previously shown, in an ideal scenario, the ICR list and the orientation of the contour are perpendicular. This means, that the angle of the velocity profile vector $\alpha_{\mathrm{CS}}$ and the orientation of the contour $\alpha_{\mathrm{c}}$ are the same, which means

$$
\alpha_{\mathrm{CS}}-\alpha_{\mathrm{c}}=0 .
$$

It should be noted, that for the simple contour construction method used here, the orientation has point symmetry around the contour's center point, assuming the long side of the constructed contour is interpreted as the length of the contour. Therefore, the contour angle has to be mapped to $\alpha_{\mathrm{c}} \in\left[-90^{\circ}, 90^{\circ}\right]$ for Eq. 3 to hold true.

Because neither the retrieved velocity profile parameters $C$ and $S$ (and thus by proxy the ICR list angle), nor the constructed OBB describing the contour of the vehicle are ideal, a maximum angle difference $\alpha_{\mathrm{d}, \max }$ has to be allowed in both directions, changing Eq. 3 to

$$
\left|\alpha_{\mathrm{CS}}-\alpha_{\mathrm{c}}\right|=\alpha_{\mathrm{d}} \leq \alpha_{\mathrm{d}, \max } .
$$

If Eq. 4 holds true for the current measurement, a linear motion is assumed and the velocity profile parameters $C$ and $S$ are directly assigned to the velocity vectors $v_{\mathrm{x}}$ and $v_{\mathrm{y}}$. Here, a maximum angle deviation of $\alpha_{\mathrm{d}, \max }=5^{\circ}$ is chosen, which has proven to be a good trade-off between stable linear motion detection and still accurate low yaw rate estimation.

If Eq. 4 does not hold true, the measurement is considered for possible nonlinear motion. To check for this, the ICR list is first constructed. Again, because of nonidealities, the detectable absolute yaw rate is restricted to $|\omega| \in\left[\omega_{\min }, \omega_{\max }\right]$, with $\omega_{\min }=5^{\circ} / \mathrm{s}$ and $\omega_{\max }=70^{\circ} / \mathrm{s}$ respectively.

This leads to a two-parted ICR list, whose starting and end points are given by Eqs. 5 and 6 .

$$
\begin{aligned}
& \left(x_{\mathrm{ICR}, \min \pm}, y_{\mathrm{ICR}, \min \pm}\right)=\left(-\frac{S}{ \pm \omega_{\max }}, \frac{C}{ \pm \omega_{\max }}\right), \\
& \left(x_{\mathrm{ICR}, \max \pm}, y_{\mathrm{ICR}, \max \pm}\right)=\left(-\frac{S}{ \pm \omega_{\min }}, \frac{C}{ \pm \omega_{\min }}\right) .
\end{aligned}
$$

Then, by projecting the reference point onto the ICR lists, a solution for $\mathbf{P}_{\mathrm{ICR}}$ can be estimated and thus an estimation for the yaw rate $\omega$.

The full subroutine for the extraction of the actual motion parameters is shown in Fig. 3.

Before any of the steps for the possible nonlinear processing are taken, the contour first gets stabilized. This is done by taking the target points used to construct the OBB and filtering out the ones that are not within the bulk motion corridor of the VPA. If the remaining target points are sufficient to build 


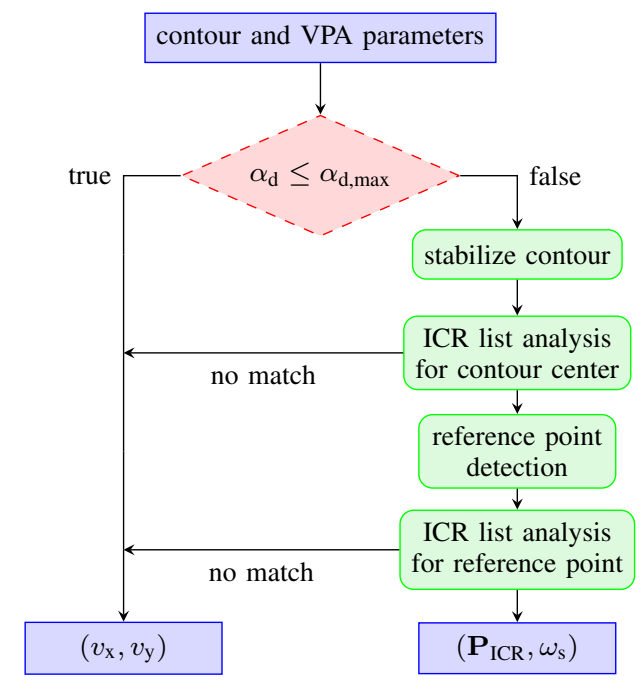

Fig. 3. Subroutine for motion parameter extraction.

a new contour, the resulting bounding box is more aligned with the contour, as there are less clutter points in the cluster. Otherwise the velocity profile parameters $C$ and $S$ are assumed to be not trustworthy and a motion estimation is not attempted.

Fig. 4 shows the extraction of the ICR from the contour and the ICR list. For a good estimation result, using the correct reference point is highly important. Ideally, the reference point is located on the rear axle $\left(R_{\text {correct }}\right)$, which then yields the correct ICR $\mathbf{P}_{\text {ICR,correct }}$ and as a result the correct yaw rate $\omega$.

To correctly set the reference point, the moving direction of the vehicle and the offset of the reference point to the backside of the vehicle have to be known. The offset has be be assumed based upon classification or by trying to identify the wheel positions [7], or - in this case - via a priori knowledge. For nonlinear motion, the information about the moving direction cannot be directly gained, without first knowing the turning direction and the segment of the turn.

Therefore, a first ICR list analysis is made by trying to project the geometrical center of the stabilized contour (C) onto the ICR list to receive $\left(\mathbf{P}_{\mathrm{ICR}, \text { center }}\right)$.

For most cases, this will yield a yaw rate and ICR correct enough to identify the moving direction of the contour. For this, a most likely lateral direction $\alpha_{\text {lat }}$, depending on the yaw rate and the position of the center point $\mathrm{C}=\left(x_{\mathrm{c}}, y_{\mathrm{c}}\right)$ relative to the ICR $\mathbf{P}_{\mathrm{ICR} \text {,center }}=\left(x_{\mathrm{i}}, y_{\mathrm{i}}\right)$ are examined. For an object in front of the radar, the following relations are valid:

$$
\alpha_{\text {lat }}= \begin{cases}0^{\circ} & \text { if } \operatorname{sign}(\omega)=\operatorname{sign}\left(y_{\mathrm{i}}-y_{\mathrm{c}}\right) \\ 180^{\circ} & \text { otherwise }\end{cases}
$$

Then, a second ICR list analysis is performed, now with the correctly determined reference point. If for any of the two ICR list analyses no projection point can be found within the limits of the ICR list, determined by the starting and end points (see Eqs. 5 and 6), a linear motion is assumed instead.

This two-stage algorithm works reasonable well, as long as the ICR list does not intersect with the contour in such a way,

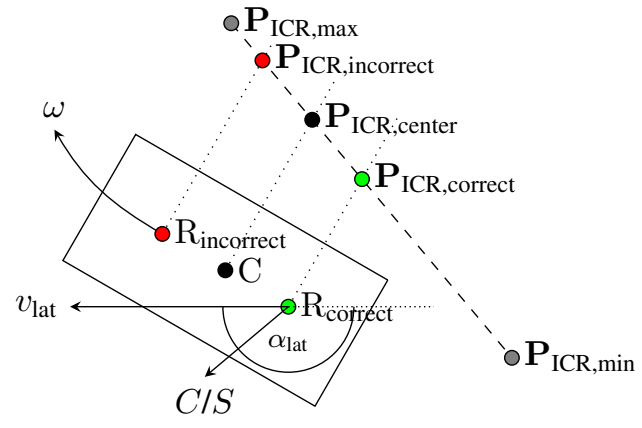

Fig. 4. Influence of the reference point location on the ICR estimation.

that the first ICR list analysis is leading to a wrongly signed yaw rate. This can happen, if the vehicle is passing right in front of the radar sensor while making a turning motion.

Also, the parameters $C$ and $S$ have to be reasonably well extracted from the velocity profile, which is harder, the smaller the angle spread of the target points. This happens for example, if the car moves away from or towards the radar sensor radially, so that only the short side of the vehicle is visible.

In these cases, the reference point can be shifted in the wrong direction along the orientation of the contour $\left(\mathrm{R}_{\text {incorrect }}\right.$ in Fig. 4) which then leads to a wrong ICR and yaw rate.

\section{MEASUREMENT DATA}

To verify the effectiveness of the proposed approach, a total of 6143 radar measurements, consisting of 2946 linear measurements with a ground truth yaw rate of $\omega_{\mathrm{gt}} \leq 5^{\circ} / \mathrm{s}$ and 3197 nonlinear measurements with a ground truth yaw rate of $5 \% / \mathrm{s}<\omega_{\mathrm{gt}} \leq 60^{\circ} / \mathrm{s}$, were analyzed. A car, equipped with an Automotive Dynamic Motion Analyzer (ADMA) for recording ground truth information, served as target vehicle.

The radar measurements were conducted with a $1 \times 8$ SIMO radar using a bandwidth of $B=2 \mathrm{GHz}$ operating in the $77 \mathrm{GHz}$ frequency band. Using a chirp sequence modulation with a high number of ramps and short ramp duration, a range resolution of $\Delta R=0.08 \mathrm{~m}$ and a velocity resolution of $\Delta v=0.11 \frac{\mathrm{m}}{\mathrm{s}}$ were achieved.

Multiple types of trajectories were included, like figure eight driving maneuvers as well as driving away from and perpendicular to the radar sensors with and without different turning maneuvers.

\section{RESULTS}

For linear motion, the yaw errors for the conventional contour approach and the angle of the extracted velocity vector $\left(v_{\mathrm{x}}, v_{\mathrm{y}}\right)$, as well as the error of the velocity vector components were evaluated.

For nonlinear motion, the error of the estimated yaw rate $\omega$ was evaluated. Also the rate of correct and incorrect detection of linear and nonlinear motion was analyzed.

To separate the effectiveness of the VPA process from that of the contour estimation itself, a second error was calculated, 


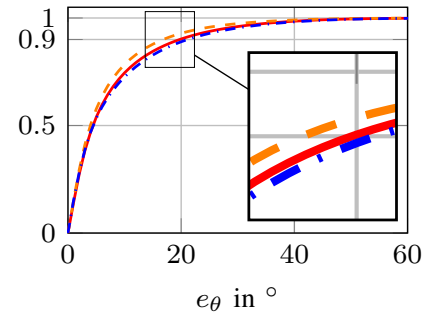

(a) Yaw errors

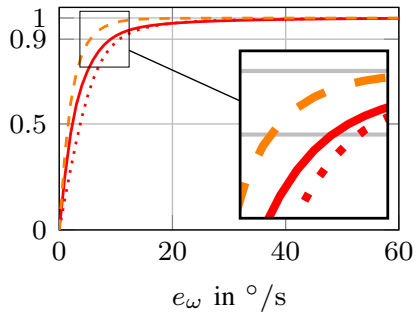

(b) Yaw rate errors.
Fig. 5. CDFs of yaw and yaw rate errors for the actual motion estimation with $\mathrm{OBB}(-)$, and ideal contour $(---)$. For the yaw error, the CDF for the conventional method is shown (-.-.). For the yaw rate error the CDF for the estimation without reference point detection is shown $(\cdots \ldots$.$) .$

using the ideal contour derived from the ground truth information for motion distinction and yaw rate estimation.

A detection rate for linear motion of $87.30 \%(95.49 \%$ for the ideal contour) was achieved. For the nonlinear motion, the detection rate was $91.52 \%(91.10 \%)$. The most errors occurred, when the vehicle starts or ends its turning motion.

For detected linear motion, table I shows the mean $(\bar{e})$, root mean square (rms), median (md), and 90th percentile $\left(e_{90}\right)$ error of the orientation $\theta$, with Fig. 5a showing its cumulative density function (CDF). A slight improvement can be seen over the conventionally constructed OBB. Mostly medium sized errors are mitigated, often caused by insufficient target points, or inconveniently clustered clutter or ground bounce points leading to badly oriented minimum area bounding boxes. The influence of a successful contour estimation is is low, meaning that an error in the velocity profile estimation is more severe for correct motion type detection than a badly constructed contour.

TABLE I

YAW ERROR FIGURES FOR THE DIFFERENT APPROACHES

\begin{tabular}{l|c|c|c|c} 
& $\overline{e_{\theta}}$ & $\mathrm{rms}\left(e_{\theta}\right)$ & $\operatorname{md}\left(e_{\theta}\right)$ & $e_{\theta, 90}$ \\
\hline Conventional Method & $7.96^{\circ}$ & $12.02^{\circ}$ & $4.42^{\circ}$ & $20.68^{\circ}$ \\
Motion Estimation (ME) & $7.60^{\circ}$ & $11.45^{\circ}$ & $4.41^{\circ}$ & $19.01^{\circ}$ \\
ME with Ideal Contour & $6.66^{\circ}$ & $10.08^{\circ}$ & $3.98^{\circ}$ & $16.51^{\circ}$
\end{tabular}

Table II shows the errors for the estimated yaw rate $\omega$ for detected nonlinear motion, with the CDF being plotted in Fig. 5b. A good estimation can be made, especially with an ideal contour. With an estimated contour, the yaw rate estimation still works well, although with significantly higher errors. The positive influence of the two-stage reference point detection is clearly visible for the small to medium errors.

TABLE II

YAW RATE ERROR FIGURES FOR THE MOTION ESTIMATION ALGORITHM.

\begin{tabular}{l|c|c|c|c} 
& $\overline{e_{\omega}}$ & $\operatorname{rms}\left(e_{\omega}\right)$ & $\operatorname{md}\left(e_{\omega}\right)$ & $e_{\omega, 90}$ \\
\hline With Center Point & $5.12 \% / \mathrm{s}$ & $7.74 \% / \mathrm{s}$ & $3.73 \% / \mathrm{s}$ & $10.32 \% \mathrm{~s}$ \\
With Reference Point & $4.07 \% / \mathrm{s}$ & $7.26 \% \mathrm{~s}$ & $2.30 \% \mathrm{~s}$ & $8.57 \% / \mathrm{s}$ \\
With Ideal Contour & $2.31 \% / \mathrm{s}$ & $3.48 \% \mathrm{~s}$ & $1.55 \% / \mathrm{s}$ & $5.27 \% / \mathrm{s}$
\end{tabular}

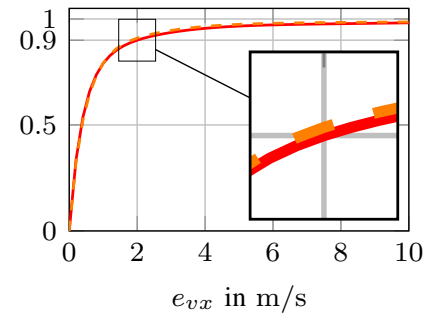

(a) $v_{x}$ errors.

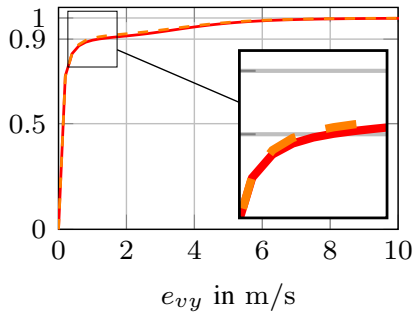

(b) $v_{y}$ errors.
Fig. 6. CDFs of velocity errors for the actual motion estimation ( - ), and for the actual motion estimation for an ideal contour $(---)$.

The errors for the estimation of the velocity vectors are shown in table III, the CDFs in Figs. 6a and 6b. Similar to the yaw error, the influence of the contour estimation is negligible. Especially the velocity vector in $y$-direction suffers from medium to high errors.

TABLE III

VELOCITY ERROR FIGURES FOR THE MOTION ESTIMATION ALGORITHM.

\begin{tabular}{l|c|c|c|c} 
& $\overline{e_{v}}$ & $\mathrm{rms}\left(e_{v}\right)$ & $\operatorname{md}\left(e_{v}\right)$ & $e_{v, 90}$ \\
\hline Motion Estimation $v_{\mathrm{x}}$ & $0.97 \mathrm{~m} / \mathrm{s}$ & $2.48 \mathrm{~m} / \mathrm{s}$ & $0.36 \mathrm{~m} / \mathrm{s}$ & $1.92 \mathrm{~m} / \mathrm{s}$ \\
Ideal Contour $v_{\mathrm{x}}$ & $0.97 \mathrm{~m} / \mathrm{s}$ & $2.97 \mathrm{~m} / \mathrm{s}$ & $0.36 \mathrm{~m} / \mathrm{s}$ & $1.75 \mathrm{~m} / \mathrm{s}$ \\
Motion Estimation $v_{\mathrm{y}}$ & $0.49 \mathrm{~m} / \mathrm{s}$ & $1.29 \mathrm{~m} / \mathrm{s}$ & $0.08 \mathrm{~m} / \mathrm{s}$ & $1.11 \mathrm{~m} / \mathrm{s}$ \\
Ideal Contour $v_{\mathrm{y}}$ & $0.45 \mathrm{~m} / \mathrm{s}$ & $1.19 \mathrm{~m} / \mathrm{s}$ & $0.08 \mathrm{~m} / \mathrm{s}$ & $0.82 \mathrm{~m} / \mathrm{s}$
\end{tabular}

\section{CONCLUSION}

The presented algorithm is able to estimate the actual motion parameters instantaneously with a single radar sensor with only small errors, especially for the yaw rate, which profits from a correctly chosen reference point. To correctly distinguish linear from nonlinear motion and for low estimation errors, the quality of the estimated contour is crucial.

\section{REFERENCES}

[1] D. Kellner, M. Barjenbruch, J. Klappstein, J. Dickmann, and K. Dietmayer, "Instantaneous Full-Motion Estimation of Arbitrary Objects using Dual Doppler Radar," in IEEE Intelligent Vehicles Symposium Proceedings, Jun. 2014, pp. 324-329.

[2] D. Kellner, M. Barjenbruch, K. Dietmayer, J. Klappstein, and J. Dickmann, "Instantaneous Lateral Velocity Estimation of a Vehicle using Doppler Radar," in Proceedings of the 16th International Conference on Information Fusion, Jul. 2013, pp. 877-884.

[3] J. Gunnarsson, L. Svensson, L. Danielsson, and F. Bengtsson, "Tracking Vehicles using Radar Detections," in 2007 IEEE Intelligent Vehicles Symposium, Jun. 2007, pp. 296-302.

[4] C. Knill, A. Scheel, and K. Dietmayer, "A Direct Scattering Model for Tracking Vehicles with High-Resolution Radars," in IEEE Intelligent Vehicles Symposium (IV), Jun. 2016, pp. 298-303.

[5] F. Roos, D. Kellner, J. Dickmann, and C. Waldschmidt, "Reliable Orientation Estimation of Vehicles in High-Resolution Radar Images," IEEE Transactions on Microwave Theory and Techniques, vol. 64, no. 9, pp. 2986-2993, Sep. 2016.

[6] J. Schlichenmaier, N. Selvaraj, M. Stolz, and C. Waldschmidt, "Template Matching for Radar-based Orientation and Position Estimation in Automotive Scenarios," in IEEE MTT-S International Conference on Microwaves for Intelligent Mobility (ICMIM), Mar. 2017, pp. 95-98.

[7] D. Kellner, M. Barjenbruch, J. Klappstein, J. Dickmann, and K. Dietmayer, "Wheel Extraction based on Micro Doppler Distribution using High-Resolution Radar," in IEEE MTT-S International Conference on Microwaves for Intelligent Mobility (ICMIM), Apr. 2015, pp. 1-4. 